\title{
Los predictores motores de la deficiencia de las funciones ejecutivas en pacientes con Enfermedad de Alzheimer*
}

Predictors engines for decline of executive functions in the elderly with Alzheimer's Disease

DOI: 10.11144/Javeriana.upsy15-5.pmdf
Recepción: 15 Diciembre 2015 | Aprobación: 16 Noviembre 2016

Renata Valle Pedroso ${ }^{a}$

University State of São Paulo, Brasil

Danilla Icassatti Corazza

University State of São Paulo, Brasil

Carla Andreza de Almeida

Andreatto

University State of São Paulo, Brasil

Julimara Gomes dos Santos

University Vale do Rio Doce, Brasil

Angelica Miki Stein

University State of São Paulo, Brasil

Oscar Gutiérrez Huamaní

University State of São Paulo, Brasil

José Luiz Riani Costa

University State of São Paulo, Brasil

Ruth Ferreira Santos-Galduròz

University Federal of ABC - UFABC, Brasil

\begin{tabular}{l}
\hline aCorresponding author. \\
re.pedroso@hotmail.com
\end{tabular}

Para citar este artículo: Valle Pedroso, R., Icassatti, C. D., Andreatto, C. A., Gomes dos Santos, J., Stein, A. M., ... \& Santos Galduróz, R. F. (2016). Los predictores motores de la deficiencia de las funciones ejecutivas en pacientes con Enfermedad de Alzheimer. Universitas Psychologica, 15(5). http:// dx.doi.org/10.11144/Javeriana.upsy15-5.pmdf

\section{RESUMEN}

El objetivo de esta investigación fue investigar las relaciones entre funciones ejecutivas y capacidad funcional y determinar los predictores motores de la deficiencia de las funciones ejecutivas en ancianos con enfermedad de Alzheimer (EA). 24 ancianos con EA fueron evaluados para estimar las funciones ejecutivas y los predictores motores. Se utilizó el análisis estadístico descriptivo, correlación de Pearson y regresión lineal múltiple. Las funciones ejecutivas se relacionaron con la flexibilidad $(\mathrm{r}=$ $0.54)$, equilibrio $(r=0.48)$, agilidad y movilidad $(r=-0.41)$. El análisis de regresión apuntó equilibrio y flexibilidad como predictores motores de la deficiencia de las funciones ejecutivas. El prejuicio del equilibrio y la flexibilidad pueden predecir los prejuicios en funciones ejecutivas de ancianos con EA.

\section{Palabras clave}

Enfermedad de Alzheimer; Capacidad Funcional; Funciones Ejecutivas; Envejecimiento; Deterioro Cognitivo; Motor.

\section{ABSTRACT}

The objective of this paper was investigate the relationship between executive function and functional capacity and determine predictors engines for decline of executive functions in elderly patients with AD. A total of 24 elderly patients with EA were evaluated to estimate predictors 
Renata Valle Pedroso, Danilla Icassatti Corazza, Carla Andreza de Almeida Andreatto, Julimara Gomes dos Santos, Et al.

engines. Descriptive statistical analysis, Pearson Correlation and multiple linear regressions was used. Executive functions related to the flexibility $(\mathrm{r}=0.54)$, static and dynamic balance $(r=0.48)$ and with the agility and mobility $(r=-0.41)$. Regression analysis pointed to balance and flexibility as predictors engines deficiency executive functions. The decline of balance and flexibility can predict the prejudice of the executive functions of elderly with AD.

Keywords

Alzheimer's Disease; Functional Capacity; Executive Functions; Aging; Cognitive Impairment; Motor.

\section{Introducción}

Como consecuencia de la muerte neuronal, los pacientes con enfermedad de Alzheimer (EA) presentan, luego en la fase inicial de la enfermedad, una serie de alteraciones cognitivas, entre ellas la pérdida de la funciones ejecutivas (FE), que son responsables por la capacidad de planeamiento mental, organización, secuencia en las tareas y abstracción (Freitas, 2006; Nitrini et al., 2005; Yaari \& Bloom, 2007).

Las FE son procesos extremadamente complejos y son constantemente requeridos durante la mayoría de las actividades del diario vivir, por lo que, todas estas pérdidas hacen con que el individuo se torne cada vez menos independiente y menos autónomo. Pues, como descrito por Royall et al. (2002), la autonomía está directamente relacionada a un buen funcionamiento ejecutivo.

Además de las alteraciones cognitivas, el paciente con EA, también presenta un perjuicio en los componentes de la capacidad funcional, como la agilidad, equilibrio, flexibilidad, fuerza, resistencia aerobia y coordinación motora. Esos disturbios motores en la EA eran comúnmente descritos en las fases más avanzados de la enfermedad, por esto, estudios recientes, relatan que mismo en fases iniciales ocurren tales alteraciones (Fuentes, 2008; Perry \& Hodges, 2000).

El estudio de Eggermont et al. (2010) constató que los ancianos en la fase leve de la EA presentan perjuicios en el equilibrio y en la movilidad funcional cuando comparados a los ancianos cognitivamente preservados.
Esta diferencia entre grupos también fueron encontradas en otros estudios en los demás componentes de la capacidad funcional, como fuerza y coordinación motora (Kato-Narita, Nitrini \& Radanovic, 2011).

Una posible explicación para las alteraciones en estos componentes de la capacidad funcional en acianos con EA puede ser la incidencia de los perjuicios cognitivos resultantes de la enfermedad. En este contexto, nuevas investigaciones sobre esta relación son importantes, una vez que todas las alteraciones cognitivas y motoras pueden perjudicar la realización de las actividades del diario vivir.

De esta forma, el objetivo del presente estudio fue investigar las posibles relaciones entre el perjuicio de las FE y las alteraciones motoras, y además, estudiar los componentes motores como posibles predictores de la pérdida de las funciones ejecutivas de los ancianos en la fase leve de la enfermedad de Alzheimer.

\section{Método y procedimiento}

\section{Pacientes}

La muestra fue compuesta por 24 ancianos con diagnóstico clínico de probable EA, con 76,9 \pm 5,3 años de promedio de edad, los cuales fueron reclutados en el Programa de Cinesioterapia Funcional e Cognitiva em Idosos com Doença de Alzheimer (PRO-CDA), que es un proyecto de extensión ofrecido por el Departamento de Educación Física del Instituto de Biociencias de la Universidad Estatal Paulita "Júlio de Mesquita Filho" (UNESP), del campus de Río Claro (Garuffi et al., 2011).

Como criterio de inclusión, los ancianos debían tener el diagnóstico probable de EA de acuerdo con el Manual Diagnóstico y Estadístico de Trastornos Mentales (DSM-IV) (APA, 2000), estar en la fase leve de la enfermedad, de acuerdo con la Clinical Dementia Rating (CDR) (Montaño \& Ramos, 2005; Morris, 1993), presentar ambulación preservada y no presentar otras condiciones neuropsiquiatrías. 


\section{Aspectos Éticos}

El presente estudio fue aprobado por el Comité de Ética de Investigación en Seres Humanos del Instituto de Biociencias de la Universidad Estatal Paulista (UNESP) de Río Claro (Protocolo $\mathrm{n}^{\mathrm{o}}$ 3174) y fue obtenido un consentimiento informado de cada participante.

\section{Procedimiento}

Los participantes hicieron su primera visita al laboratorio, para la constatación del diagnóstico y de la fase de la EA. En un segundo momento fueron programadas las evaluaciones cognitivas y motoras. Todas las evaluaciones fueron realizadas por un equipo de profesionales entrenados para la aplicación de los test y cuestionarios. El protocolo de evaluación fue compuesto por los siguientes instrumentos:

\section{Entrevista Estructurada}

Para obtener datos socio-demográficos e informaciones sobre el paciente, como el tiempo de cuidado, tareas con el paciente, condición clínica general, enfermedad y medicamentos.

\section{Mini-Examen del Estado Mental (MEEM)}

Es un instrumento que consta de preguntas agrupadas en siete categorías, cada una diseñada con el objetivo de evaluar las funciones cognitivas específicas. Ellas son: la orientación del tiempo, orientación del lugar, memoria, atención y cálculo, lenguaje y visión constructiva. La puntuación MMSE va de 0 a 30 puntos, y los valores más bajos indican un posible deterioro cognitivo (Folstein, Folstein \& Mchugh, 1975).

\section{Clinical Dementia Rating (CDR)}

El principal objetivo del CDR es clasificar la gravedad (o fase) de la demencia. Este instrumento se divide en seis categorías de comportamiento cognitivo: memoria, orientación, juicio o resolución de problemas, asuntos de la comunidad, aficiones en el hogar y el cuidado personal. Cada una de estas seis categorías deberían clasificarse como: cero (sin cambios); 0,5 (demencia cuestionable); 1 (demencia leve); 2 (demencia moderada); y 3 (demencia grave) (Montaño \& Ramos, 2005; Morris, 1993).

\section{Test de Diseño del Reloj (TDR)}

El test incluye la obligación de elaborar un reloj con la inclusión de indicadores que califican 02:45 a.m. Esta prueba evalúa las funciones ejecutivas (planificación, secuencia lógica de ejecución), organización visualespacial, praxis viso-constructiva, coordinación psicomotriz (Sunderland et al., 1989). El TDR ha sido traducido, adaptado y validado en Brasil por Atalaia-Silva y Lourenço (2008).

\section{Escala de Depresión Geriátrica (GDS - 30)}

La escala está constituida por 30 preguntas con respuestas dicotómicas (sí/no), desarrollada para la población anciana, utilizada para cuantificar los síntomas depresivos. En Brasil, fue traducida y validada, siendo indicada sensibilidad de $100 \%$ y especificidad de $88 \%$ para el punto de corte en 9 (Yesavage et al., 1983).

\section{Cuestionario Baecke Modificado para Ancianos (CBMA)}

Evalúa el nivel de actividad física de ancianos, considerando un año de actividad en los diferentes dominios, como ocio, deportes y tareas del hogar. Este cuestionario presenta buena validad discriminante, de contenido y también buena estabilidad (Voorrips et al., 1991). 
Renata Valle Pedroso, Danilla Icassatti Corazza, Carla Andreza de Almeida Andreatto, Julimara Gomes dos Santos, Et al.

\section{Test de Caminata de 6 minutos}

El objetivo principal es verificar la resistencia cardiovascular, este test mide la mayor distancia recorrida de caminata durante seis minutos. El test es realizado entre conos posicionados a una distancia de 30 metros. El participante, inicia de un cono en dirección al otro, debe andar lo más rápido posible (sin correr o trotar), contornar el otro cono y volver, realizando ese circuito durante seis minutos. El examinador debe contar el número de vueltas entre los conos realizadas por el participante (Rikli \& Jones, 1999).

Sentarse y levantarse de la silla en 30 segundos

Evalúa la resistencia de fuerza de miembros inferiores a través del número máximo de veces que el individuo realiza la acción de sentarse y levantarse de la silla, sin apoyo, en 30 segundos (Rikli \& Jones, 1999).

\section{Flexión del codo en 30 segundos (AAHPERD)}

Evalúa la resistencia de fuerza de miembros superiores a través del número máximo de veces que el individuo realiza las flexiones el codo asegurando pesas de $1,814 \mathrm{~kg}$ (para mujeres) y $3,628 \mathrm{~kg}$ (para hombres), en un periodo de 30 segundos (Osness et al., 1990).

\section{Banco de Wells}

Para medir la flexibilidad con el uso del protocolo "Sentar y Alcanzar" de Wells y Dilon (1952), en que el individuo debe sentarse de frente para el banco, colocando los pies en el apoyo con las rodillas extendidas; extiende el brazo y sobrepone una mano a la otra y las lleva hacia frente tocando la cinta métrica lo más lejos y largo que pueda, sin flexionar las rodillas. Los valores pueden variar, al "no conseguir alcanzar" hasta $56 \mathrm{~cm}$, que es el valor máximo que la cinta métrica proporciona en el test.

\section{Escala de Equilibrio Funcional de Berg (EEFB)}

La escala está compuesta por 14 ítems envolviendo tareas funcionales específicas en diferentes bases de apoyo. Cada tarea es subdividida y puntuada de acuerdo con el grado de dificultad. El escore varía entre cero y 56, con puntos inferiores caracterizando un mayor riesgo de caídas (Berg, Wood-Dauphinee, Williams \& Maki, 1992).

\section{Timed Up-and-Go (TUG)}

Test de fácil aplicación que evalúa la movilidad funcional básica. En él es analizado el tiempo gastado por el individuo para levantarse de una silla, andar por una distancia de tres metros y retornar a la silla, así como el número de pasos necesarios para la ejecución de la actividad. Valores más altos de tiempo y número de pasos representa mayor riesgo de caída (Podsiadlo \& Richardson, 1991).

\section{Análisis estadístico}

Para el análisis de los resultados se realizó: a) estadística descriptiva; b) test de normalidad por medio del test de Shapiro Wilk; c) padronización de los datos utilizándose el escore Z; d) determinación del grado de asociación, utilizándose el coeficiente de correlación de Pearson para la variable dependiente (funciones ejecutivas), y las variables independientes (componentes motores de la capacidad funcional); e) test de regresión múltiple paso a paso (stepwise). En todos los casos, fue adoptado un nivel de significación de $5 \%$.

\section{Resultados}

Todas las características se presentan en la tabla 1 en promedio y desviación estándar (DE). En la tabla 2 pueden ser observados el promedio y DE para cada variable dependiente e independiente. Los coeficientes de correlación 
de Pearson mostraron asociaciones significativas entre las funciones ejecutivas y los componentes de la capacidad funcional, presentadas en la tabla 3. Para las funciones ejecutivas, fue verificada la correlación directa y moderada con la flexibilidad (Wells) $(\mathrm{r}=0.54)$ y el equilibrio estático y dinámico (EEFB) $(\mathrm{r}=0.48)$, y una correlación inversa y baja con la agilidad y movilidad $(\mathrm{r}=$ -0.41) del paciente con EA.

\section{TABLA 1}

Características sociodemográficas de los ancianos con enfermedad de Alzheimer, expresados en promedio y desviación estándar (DE)

\begin{tabular}{lc}
\hline Caracterización de la muestra & Promedio y DE \\
\hline Edad (años) & $76.9 \pm 5.3$ \\
Escolaridad (años) & $5.0 \pm 4.1$ \\
MEEM (puntos) & $19.8 \pm 4.5$ \\
GDS-30 (puntos) & $5.9 \pm 4.6$ \\
CBMA (puntos) & $2.4 \pm 1.7$ \\
\hline
\end{tabular}

MEEM: Mini-Examen del Estado Mental, EA: Enfermedad de Alzheimer, GDS-30: Escala de Depresión Geriátrica, CBMA: Cuestionario de Baecke Modificado para Ancianos; DE: desviación estándar. Fuente: elaboración propia

\section{TABLA 2}

Promedios y desviación estándar (DE) para las funciones ejecutivas (variable dependiente) y los componentes motores de la capacidad funcional (variables independientes)

\begin{tabular}{lcc}
\hline Dominio Evaluado & Instrumentos & Promedio y DE \\
\hline Funciones Ejecutivas & TDR (puntaje) & $1.4 \pm 0.9$ \\
\hline Fuerza de Miembros Inferiores & Resistencia MI (Rep.) & $11.2 \pm 2.9$ \\
\hline Fuerza de Miembros Superiores & Resistencia MS (Rep.) & $11.7 \pm 5.1$ \\
\hline Flexibilidad & Banco de Wells (cm) & $18.5 \pm 8.6$ \\
\hline Agilidad y Movilidad & TUG Tiempo (s) & $8.6 \pm 1.9$ \\
\cline { 2 - 3 } & TUG Pasos & $16.5 \pm 4.1$ \\
\hline Equilibrio & EEFB (puntos) & $51.8 \pm 2.7$ \\
\hline Resistencia Aerobia & Teste dos 6 minutos (m) & $395.2 \pm 96.0$ \\
\hline
\end{tabular}

TDR: Test de Diseño del Reloj; MI:

Miembros Inferiores; MS: Miembros Superiores; TUG: "Timed Up and Go"; Rep: repeticiones; DE: desviación estándar. Fuente: elaboración propia

\section{TABLA 3}

Coeficiente de correlación de Pearson ( $r$ y significación (p) entre las funciones ejecutivas (variable dependiente) y los componentes de la capacidad funcional (variables independientes)

\begin{tabular}{lcc}
\hline Componentes Motores & $\boldsymbol{r}$ & $\boldsymbol{p}$ \\
\hline Fuerza de Miembros Inferiores & 0.16 & 0.46 \\
\hline Fuerza de Miembros Superiores & 0.22 & 0.30 \\
\hline Flexibilidad & 0.54 & $<0.01$ \\
\hline Agilidad y Movilidad & 0.41 & 0.43 \\
\cline { 2 - 3 } & 0.26 & 0.21 \\
\hline Equilibrio & 0.48 & 0.01 \\
\hline Resistencia Aerobia & 0.40 & 0.51 \\
\hline
\end{tabular}

Fuente: elaboración propia

El resultado de la regresión múltiple paso a paso (stepwise) confirmó la asociación de las funciones ejecutivas con algunos componentes de la capacidad funcional, como la flexibilidad $(\mathrm{p}=0.02 ; \#=0.46)$ y equilibrio estático y dinámico ( $\mathrm{p}=0.04 ; \#=0.39$ ) de los pacientes con EA. Realizado el análisis de regresión múltiple paso a paso (stepwise), el modelo de regresión fue: Funciones Ejecutivas $=-6,93+(0,46 \times$ flexibilidad $)+(0,39 \times$ equilibrio estático y dinámico). En la ecuación de regresión citada, $-6,93$ es una constante. De esta forma, el perjuicio de la flexibilidad y del equilibrio estático y dinámico pueden predecir el perjuicio de las funciones ejecutivas en $0.46 \mathrm{y}$ 0.39 veces, respectivamente. El ajuste del modelo fue evaluado por medio de un análisis de residuos.

\section{Discusión}

Los datos del presente estudio sugieren una asociación entre las variables analizadas, respondiendo al objetivo de investigar las relaciones entre funciones ejecutivas y los componentes motores de la capacidad funcional de pacientes con EA. También pudo ser observado que las asociaciones revelan, entre todos los componentes motores, que aquellos que se relacionan con el prejuicio de las funciones ejecutivas son el equilibrio (estático y dinámico) y la flexibilidad, respondiendo así a los objetivos propuestos. 
Renata Valle Pedroso, Danilla Icassatti Corazza, Carla Andreza de Almeida Andreatto, Julimara Gomes Dos Santos, Et al.

El equilibrio es un componente fundamental para la independencia del individuo, pues, es responsable por la mantención de la posición del cuerpo en el espacio, realizada a través de la integración de tareas motoras por medio de varias informaciones sensoriales, planeamiento y ejecución del movimiento (Horak \& MacPherson, 1993). Los individuos con la EA tienden a presentar pérdida en el procesamiento de las informaciones sensoriales y deficiencias de las funciones ejecutivas, lo que puede agravar el equilibrio, aumentando el riesgo de caídas en esta población (Kluger et al., 1997). Así, el $60 \%$ de los pacientes ancianos con declino cognitivo sufre caídas, dos veces más de que aquellos cognitivamente preservados (Davis, Hsiung \& Ambrose TL, 2011). Por tanto, el presente estudio sugiere que el déficit cognitivo puede interferir en el control del equilibrio de ancianos con EA en la fase leve de la enfermedad. Todas estas alteraciones están asociadas con la pérdida de independencia y calidad de vida.

Nuestros resultados corroboran los hallazgos de un estudio conducido por Arcoverde et al. (2008). A pesar de no tener investigado específicamente las funciones ejecutivas, los autores demostraron en su estudio que la eficiencia cognitiva global del anciano con EA, medida a través del MEEM, presentaba relación con el equilibrio, sugiriendo así, que los ancianos con EA más preservados cognitivamente presentan mejor equilibrio, comparados a los menos preservados. Por ello, los autores se limitaron a estudiar el equilibrio y no evaluaron los demás componentes de la capacidad funcional.

En este estudio, además del equilibrio haber sido apuntado como un predictor de alteraciones en las funciones ejecutivas, la flexibilidad también mostró ser un componente importante. No fue encontrado en la literatura estudios que investigasen la relación entre funciones ejecutivas y flexibilidad, pero estudios apuntan que la flexibilidad parece estar perjudicada en la EA, luego de las fases iniciales (Zidan et al., 2012).

La flexibilidad representa un importante componente de la capacidad funcional, pues un buen nivel de flexibilidad está relacionado a una mejor movilidad, lo que se refleja en un satisfactorio desempeño en la realización de las actividades del día a día (Farinatti, 2002).

De esta forma, el presente estudio indica que, además de haber una asociación entre cognición y aspectos motores, menores niveles de equilibrio y flexibilidad pueden predecir la deficiencia de funciones ejecutivas, y tornarse importante para investigar cuales serían los posibles mecanismos involucrados en esta relación. Estudios recientes sugieren que, los mecanismos por los cuales ancianos con EA también presentan alteraciones motoras pueden ser semejantes a los mecanismos de la alteración cognitiva (Burns, Galvin, Roe, Morris \& McKeel, 2005; Schneider et al., 2006).

Estudios post-mortem en pacientes con EA concluyeron que la neuropatología de la enfermedad, como el acumulo de proteínas \#-amiloide y la presencia de enmarañados fibrilares, también están presentes en la corteza motora primaria y complementares, estriado y sustancia nigra, los cuales son responsables por los movimientos del cuerpo (Burns et al., 2005; Schneider et al., 2006). En conjunto, estos datos sugieren que la neuropatología de la EA está presente en las regiones corticales y subcorticales pudiendo desarrollar no solamente la pérdida cognitiva, pero también las alteraciones motoras.

Los hallazgos del presente estudio son de gran relevancia, una vez que indican los predictores motores de la pérdida de las funciones ejecutivas y puede ser útil para ayudar a esclarecer la relación entre cognición y capacidad funcional. Zidan et al. (2012) mostraron que tanto el deterioro cognitivo cuanto motor en la EA aumentan gradualmente de acuerdo con el avance de la enfermedad. Por tanto, estudios que investigan alteraciones cognitivas y motoras en la fase inicial de la enfermedad son importantes, pues ayudan en la construcción de estrategias de prevención y tratamiento, a fin de proporcionar más calidad de vida a esos ancianos.

Es importante resaltar la originalidad del estudio, una vez que estudios que evalúan las alteraciones motoras en la EA aún son escasos, lo que dificulta una discusión más extensa.

| Universitas Psychologica | V. I5 | No. 5 | 2016 | 
A respecto de su singularidad y pertinencia, este estudio presenta limitaciones que deben ser consideradas. El hecho de ser un estudio de corte transversal no permite que se establezca una relación de causa, más entendemos que el análisis de regresión permite una asociación relevante.

Otro aspecto que debe ser ponderado es que los test motores exigen muchas instrucciones, lo que requiere una buena comprensión por parte de los ancianos con EA, que están siendo evaluados. Por tanto, la ejecución de los test motores puede estar directamente asociada al nivel de las funciones cognitivas y no solamente de la capacidad funcional.

Siendo así, se puede concluir que hay relación entre funciones ejecutivas y la capacidad funcional de ancianos con EA, y que las variables apuntadas como predictores motores de la pérdida de las funciones ejecutivas fueron el equilibrio y la flexibilidad, es decir, la presencia de alteraciones del equilibrio y la flexibilidad pueden predecir la presencia de una pérdida en las funciones ejecutivas. Se sugiere más estudios para esclarecer aún más esta relación en ancianos en la fase leve de la enfermedad y que, además, investiguen si esos fenómenos de asociación también están presentes en ancianos con deficiencia cognitiva leve.

\section{Agradecimiento}

Los autores expresan su agradecimiento al Laboratório de Atividade Física e Envelhecimento (LAFE), del Departamento de Educação Física de la Universidade Estadual Paulista (UNESP, Río Claro, SP); el Programa de Cinesioterapia Funcional e Cognitiva em Idosos com Doença de Alzheimer (PRO-CDA); el apoyo financiero de FAPESP (Fundação de Amparo à Pesquisa do Estado de São Paulo, Proceso n. 2011/03451-8) y Coordenação de Aperfeiçoamento de Pessoal de Nível Superior (CAPES).

\section{Referencias}

American Psychiatric Association (APA). (2000). Diagnostic and statistical manual of mental disorders. Washington, DC: APA.

Arcoverde, C., Deslandes, A., Rangel, A., Rangel, A., Pavão, R., ... Laks, J. (2008). Role of Physical Activity on the Maintenance of Cognition and Activities of Daily Living in Elderly with Alzheimer's Disease. Arq Neuropsiquiatr, 66, 323-327.

Atalaia-Silva, K.C., \& Lourenço, R.A. (2008). Tradução, adaptação e validação de construto do Teste do Relógio aplicado entre idosos no Brasil. Rev Saude Publica, 42, 930-937.

Berg, K., Wood-Dauphinée, S., Williams, J.I., \& Maki, B. (1992). Measuring balance in the elderly: validation an instrument. Can J Public Health, 83, Supll2, S7-11.

Burns, J.M., Galvin, J.E., Roe, C.M., Morris, J.C., \& McKeel, D.W. (2005). The pathology of the substantia nigra in Alzheimer disease with extrapyramidal signs. Neurology, 64, 1397-1403.

Davis, J.C., Hsiung, G.Y.R, \& Ambrose, T.L. (2011). Challenges moving forward with economic evaluations of exercise intervention strategies aimed at combating cognitive impairment and dementia. $\mathrm{Br} \mathrm{J}$ Sports Med, 45, 470-472.

Eggermont, L.H., Gavett, B.E., Volkers, K.M., Blankevoort, C.G., Scherder, E.J., ... Stern, R.A. (2010). Lower-Extremity Function in Cognitively Healthy Aging, Mild Cognitive Impairment, and Alzheimer's Disease. Archives of Physical Medicine and Rehabilitation, 91, 584-588.

Farinatti, P.T.V. (2008). Envelhecimento, promoção da saúde e exercício: bases teóricas e metodológicas. Barueri - São Paulo: Manole.

Folstein, M.F., Folstein, S.E., \& Mchugh, P.R. (1975). Mini Mental State: A practical method for grading the cognitive state of patients for clinician. J Psychiatr Res, 12, 189-98.

Freitas, E.V. Tratado de geriatria e gerontologia. Rio de Janeiro. Guanabara Koogan, 2006. 
Renata Valle Pedroso, Danilla Icassatti Corazza, Carla Andreza de Almeida Andreatto, Julimara Gomes dos Santos, Et al.

Fuentes, G.P. (2008). Funcionalidad y demencia. Revista HCCH,19, 324-329.

Garuffi, M., Gobbi, S., Hernandez, S.S.S., Vital, T.M., Stein, A.M., Pedroso, R.V., ... Stella, F. (2011) Atividade Física para promoção da saúde de idosos com doença de Alzheimer e seus cuidadores. Rev. Bras. Ativ. Fis Saúde, $16,80-83$.

Horak, F.B., \& MacPherson, J.M. (1996). Postural orientation and equilibrium. In: Rowell, L.B., \& Shepherd, J.T. (Eds.). Exercise: regulation and integration of multiple systems. New York: Oxford University Press, 255-292.

Kato-Narita, E.M., Nitrini, R., \& Radanovic, M. (2011). Assessment of balance in mild and moderate stages of Alzheimer's disease. Implications on falls and functional capacity. Arq Neuropsiquiatr, 69, 202-207.

Kluger, A., Gianutsus, J. G., Golomb, J., Ferris, S. H., George, A. E., Frassen, E., \& Reiberg, B. (1997). Patterns of motors impairment in normal aging, mild cognitive decline, and early Alzheimer's Disease. Journal of Gerontology, 52B, 28-39.

Montaño, M. B., \& Ramos, L. R. (2005). Validade da versão em portugués da Clinical Dementia Rating (CDR). Rev Saúde Públ, 39, 912-917.

Morris, J. (1993). The Clinical Dementia Rating (CDR): Current version and scoring rules. Neurology, 43, 2412-2414.

Nitrini, R., Caramelli, P., Bottino, C.M.C., Damasceno, B.P., Brucki, S.M.D., \& Anghinah, R. (2005). Diagnóstico de Doença de Alzheimer no Brasil, Arq Neuropsiquiatr, 63, 720-727.

Osness, W.H., Adrian, M., Clark, B., Hoeger, W., Raab, D., \& Wiswell, R. (1990). Functional Fitness Assessment for Adults Over 60 Years. The American Alliance For Health, Physical Education, Recreation and Dance. Association For Research Administration, Professional Councils, and Societies. Council On Aging and Adult Development. 1900 Association Drive. Reston, VA 22091.

Perry, R.J., \& Hodges, J.R. (2000). Relationship between functional and neuropsychological performance in early Alzheimer disease. Alzheimer Dis Assoc Disord, 14, 1-10.

Podsiadlo, D., \& Richardson, S. (1991). The "Timed Up and Go": a test of basic functional mobility for frail elderly persons. J Am Geriatr Soc, 39, 142-148.

Rikli, R.E., \& Jones, C.J. (1999) Development and validation of a functional fitness test for community-residing older adults. J Aging Phys Activ, 7, 129-161.

Royall, D.R., Lauterbach, E.C., Cummings, J.L., Reeve, A., Rummans, T.A., ... Coffey, C.E. (2002). Executive control function: a review of its promise and challenges for clinical research. A report from the Committee on Research of the American Neuropsychiatry Associations. J Neuropsychiatry Clin Neurosci, 14, 377-405.

Schneider, J.A., Li, J.L., Li, Y., Wilson, R.S., Kordower, J.H., \& Bennett, D.A. (2006). Substantia nigra tangles are related to gait impairment in older persons. Ann. Neurol, 59, 166-173.

Sunderland, T., Hill, J.L., Melow, A.M., Lawlor, B.A., Gunderland, T., Newhouse, P.A., \& Grafman, J.H. (1989). Clock drawing in Alzheimer's disease: a novel measure of dementia severity. JAGS, 37, 725-729.

Voorrips, L., Ravelli, A., Dongelmans, P., Deurenberg, P., \& Van Staveren, W.A. (1991) A physical activity questionnaire for elderly. Med Sci Sports Exerc, 23, 974-979.

Wells, K.F., \& Dillon, E.K. (1952). The Sit and Reach - A test of Back and Leg Flexibility. Res Q Exerc Sport, 23, 115-118.

Yaari, B., \& Bloom, J.C. (2007). Alzheimer's Disease . Seminars in Neurology, 27, 32-41.

Yesavage, J.A., Brink, T.L., Lum O., Huang, V., Adey, M., \& Leirer, V.O. (1983). Development and validation of a geriatric screening scale. J Psychiatr Res, 17, 37-49.

Zidan, M., Arcoverde, C., Araújo, N.B., Vasques, P., Rios, A., Laks, J., \& Deslandes, A. (2012). Alterações Motoras e Funcionais em Diferentes Estágios da Doença de Alzheimer. Revista de Psiquiatria Clínica, 39, 161-165. 


\section{Notas}

* Artículo de investigación. 